\title{
ALGUNAS CRÍTICAS A LA CONCEPCIÓN EPISTÉMICA DE LA DEMOCRACIA DELIBERATIVA DE CARLOS NINO
}

\author{
Mariano Garreta LeClercQ \\ Universidad de Buenos Aires - CONICET -CIF - Grupo de Filosofía Política
}

\begin{abstract}
Resumen
El presente artículo propone algunas objeciones contra la concepción deliberativa de la democracia desarrollada por Carlos Nino. El blanco central de las objeciones es la tesis del filósofo argentino según la cual el valor del debate democrático derivaría, fundamentalmente, de sus virtudes epistémicas, es decir, de su capacidad para elevar las probabilidades de que el sistema político tome las decisiones correctas. Se cuestiona el modo en que el autor presenta su propuesta como una forma de superar las deficiencias que presentarían las concepciones de John Rawls y Jürgen Habermas en el campo de la epistemología moral. Se intentará demostrar que el modelo de deliberación defendido por Nino no resulta aplicable a un contexto de pluralismo razonable filosófico, religioso y moral como el que resulta característico de las democracias liberales contemporáneas. Por último, se ofrece el esbozo de una concepción alternativa, práctica y moral, no epistémica, del valor de los procedimientos democráticos y de la naturaleza de la legitimidad política.
\end{abstract}

PALABRAS CLAVE: Deliberación; Justificación; Moral; Legitimidad; Liberalismo.

\begin{abstract}
This paper posits some objections against the deliberative conception of democracy developed by Carlos Nino. According to Nino, the value of democratic deliberation lies, basically, in its epistemic virtues. Public deliberation increases the probability that the political system will arrive at the correct decisions. We shall argue that the Argentinean philosopher fails to prove that his proposal is a solution to purported weaknesses in John Rawls' and Jürgen Habermas' positions in the field of moral epistemology. We shall try to demonstrate that the conception of deliberation posited by Nino cannot be applied successfully in the context of moral, philosophical or religious reasonable pluralism that characterizes liberal contemporary democracies. Finally, we offer an outline of an alternative conception - practical and moral, non epistemic- of the value of democratic procedures and about the nature of political legitimacy.
\end{abstract}

KEY WORDS: Deliberation; Justification; Morality; Legitimacy; Liberalism.

La concepción deliberativa de la democracia elaborada por Carlos Nino a fines de los años 80 y principios de los 90 no solo tiene el valor de 
haber sido una de las primeras teorías de una corriente de pensamiento político que fue ampliamente desarrollada y discutida en las décadas posteriores. Nino propone una justificación del valor de la democracia y, en particular, de la deliberación pública, sumamente ambiciosa e interesante. Espero que pueda concluirse a partir de estas páginas que a pesar de los años transcurridos todavía es sumamente fructífero examinar críticamente las tesis fundamentales de dicha propuesta.

Algunos autores han sostenido que el valor de complementar los procedimientos de toma de decisión característicos de las democracias representativas constitucionales con instancias de deliberación libre en las que todos los afectados -o sus representantes- puedan hacer oír su voz tiene un valor fundamentalmente moral, en un sentido específico: dicho proceso de deliberación previa constituiría una precondición de la legitimidad de las decisiones que finalmente sean tomadas por medio de procedimientos agregativos. La existencia de foros ampliamente inclusivos, en los que todos los afectados por los resultados de las decisiones políticas -o sus representantes- puedan hacer oír su voz, sería un requisito indispensable para tratar a los ciudadanos con igual consideración y respeto. Esta idea es, sin duda, muy plausible y posee significativa fuerza intuitiva. No creo que Nino la desestimara por completo, sin embargo, su concepción deliberativa de la democracia no le otorga un papel central, ni se apoya en ella de forma explícita o directa. La tesis central de Nino es que "el valor de la democracia reside en su naturaleza epistémica con respecto a la moralidad social". A su juicio, la democracia, "una vez hechos ciertos reparos [...] es el procedimiento más confiable para acceder al conocimiento de los principios morales [de naturaleza intersubjetiva]" (Nino 1997, p. 154). Las razones que tienen, desde esta perspectiva, los individuos para obedecer las decisiones tomadas por los regímenes políticos que se ajustan a esta descripción deriva también de ese rasgo de orden epistémico: los procedimientos democráticos disponen, al menos en el mediano o largo plazo -puede haber errores puntuales, por supuesto-, de una probabilidad mayor de permitir determinar cuáles son los principios moralmente correctos que la probabilidad que tienen los individuos de identificar por sí mismos tales principios correctos o verdaderos. Dada esta mayor probabilidad que poseería en general el procedimiento colectivo democrático para alcanzar o aproximarse a la solución moralmente correcta, aun en el caso de que un individuo o un grupo de individuos supieran con certeza que una decisión mayoritaria particular es errónea, tendrían la obligación de acatarla (Nino 1997, p. 182). Así, tanto la legitimidad política como la obligación de obedecer al Estado derivan de consideraciones epistémicas. 
Nino pensaba que, incluso reconociendo la escala y complejidad de las sociedades modernas y la necesidad de la vigencia de un sistema representativo de gobierno, resulta vital institucionalizar procesos de deliberación colectiva. Esto es, crear un contexto social e institucional en que resulte viable "maximizar las oportunidades del debate libre, reflexivo e informado y de participación de todos, para que las decisiones que se obtienen a través del procedimiento democrático alcancen el mayor grado de validez moral" (Nino 1989, p. 127).

El valor epistémico de los procedimientos democráticos deriva de su carácter deliberativo. Los presupuestos filosóficos de la propuesta de Nino son, al menos en cierto sentido, muy claros. En primer lugar, la teoría suscribe una concepción metaética cognitivista. Los juicios morales tienen el estatus de creencias susceptibles de predicación de verdad o falsedad y el conocimiento moral, aunque resultara falible y provisorio, es una meta que tiene sentido perseguir. Más específicamente, el autor sostiene una tesis ontológica acerca de la naturaleza de la verdad moral y una tesis epistemológica acerca de las condiciones más favorables para su conocimiento. La "tesis ontológica" afirma que:

La verdad moral se constituye por presupuestos formales o procedimentales de una práctica discursiva social destinada a cooperar y evitar conflictos sobre la base de la convergencia de acciones y actitudes dada por el consenso en la aceptación de principios para guiar la conducta. Entre esos presupuestos de la práctica social del discurso moral está el de que un principio es válido cuando es aceptable o no rechazable por todos en condiciones de imparcialidad, racionalidad y conocimiento de los hechos (Nino 1989, p. 104).

La "tesis epistemológica" afirma que

La discusión y la decisión intersubjetiva es el procedimiento más confiable de acceso a la verdad moral [...] ya que el intercambio de ideas y la necesidad de justificarse frente a los demás no solo amplía el conocimiento y permite detectar fallas de razonamiento sino que principalmente permite determinar la satisfacción del requisito de atención imparcial de los intereses de todos, bajo el supuesto de que no hay mejores intérpretes de los intereses de los involucrados que los mismos afectados que participan en el proceso colectivo de discusión. Sin embargo, esto no excluye que por vía de la reflexión individual alguien pueda acceder al conocimiento de las soluciones correctas, aunque hay que admitir que este método es mucho menos confiable 
sobre todo por la dificultad para representarse fielmente los intereses de los demás y ser, por lo tanto, imparcial (Nino 1989, p. 105).

Nino concibe el discurso moral como una práctica social cuya meta es obtener consenso unánime en torno de principios que permitan justificar la realización de acciones frente a conflictos prácticos concretos o la elaboración de mecanismos institucionales para enfrentar problemas recurrentes en la coordinación de la cooperación social. El autor piensa que, bajo ciertas condiciones apropiadas en las que se preserva el potencial cognitivo de la deliberación, la unanimidad es el equivalente funcional de la imparcialidad. ${ }^{1}$ Según argumenta

Si todos aquellos que pueden ser afectados por una decisión han participado en la discusión y han tenido una oportunidad igual de expresar sus intereses y justificar una solución a un conflicto, esta será muy probablemente imparcial y moralmente correcta siempre que todos la acepten libremente y sin coerción (Nino 1997, p. 166).

Nino sostiene que el discurso moral es un caso de "justicia procesal pura perfecta [...] el único criterio de validez de sus resultados es que hayan seguido sus reglas y estas aseguran esa validez" (Nino 1989, p. 127). Imaginemos un ejemplo para entender la posición de Nino. Supongamos que un utilitarista interpreta su concepción, desde una perspectiva metaética, como una posición cognitivista. ${ }^{2}$ El utilitarista afirma que el siguiente juicio moral es verdadero: "realizar la acción A que producirá el estado de cosas X es la única alternativa moralmente aceptable en el contexto particular de acción -espacial y temporal- C en que se encuentra el sujeto $\mathrm{S}$, dado que es la opción que produce la suma neta mayor de utilidad, esto es, la suma neta mayor de placer, tomando en cuenta imparcialmente a todos los afectados". Si le preguntamos al utilitarista del ejemplo por qué afirma que ese juicio es verdadero, puede respondernos que ello se debe a que la acción A efectivamente causará el estado de cosas X y que X es el estado de cosas más valioso desde una perspectiva moral que resulta posible producir en C. Es decir, que la verdad del juicio depende, en última instancia, de que el estado de cosas $\mathrm{X}$ realmente tenga la propiedad moral que se le atribuye. Esta forma de

\footnotetext{
${ }^{1}$ Véase Nino (1989, p. 109) y (1997, p. 166).

2 Como es sabido, adoptar una posición utilitarista en el plano normativo no implica necesariamente un compromiso con el cognitivismo en el metaético, pero tampoco excluye esa posibilidad.
} 
cognitivismo moral realista -y naturalista- es incompatible con la concepción de Nino. Como vimos, para Nino la verdad del juicio en cuestión-suponiendo que fuese verdadero, por supuesto- no dependerá de que $\mathrm{X}$ sea el estado de cosas más valioso que es viable realizar, sino de que dicho juicio pudiera ser objeto de consenso unánime de todos los afectados en condiciones ideales de discusión moral (imparcialidad, racionalidad, conocimiento pleno de los hechos relevantes, etc.). Esa parece la interpretación más natural de la tesis de que no hay un criterio de validez o de verdad moral externo a las reglas procesales que, bajo las condiciones ideales referidas líneas atrás, hacen que el consenso unánime sea un equivalente funcional de la imparcialidad. ${ }^{3}$

A diferencia de la "tesis ontológica", la "tesis epistemológica" defendida por Nino es, en principio, compatible con una variedad más amplia de concepciones cognitivistas y resulta, por esa misma razón, mucho menos controvertida. El diálogo, orientado al entendimiento recíproco entre sujetos con intereses divergentes, desarrollado en foros apropiados - por ejemplo, aquellos en los que los agentes involucrados tengan una honesta disposición a intentar probar a sus interlocutores, por medio de argumentos genuinos, ${ }^{4}$ que sus intereses son legítimos y no representan meramente un intento de obtener ventajas egoístas o inmerecidas-parece aumentar las probabilidades de que se produzca una mejora en la calidad epistémica de las posiciones de los involucrados, incluso si ello no redunda en el consenso unánime. Como vimos, el autor otorga, además, significativa importancia a dos tesis adicionales. En primer lugar, a la idea de que es posible, aunque poco probable, que un individuo llegue, por sí mismo, a una visión más cercana a la verdad de la favorecida por la mayoría tras un proceso deliberativo adecuado. En segundo lugar, a la idea de que cada individuo es el mejor juez de sus propios intereses.

Ahora bien, Nino no solo sostiene que la unanimidad es el equivalente funcional de la imparcialidad, sino, pasando rápidamente del terreno moral al político, que una democracia deliberativa es el sucedáneo del discurso moral. Como vimos, si las instituciones y procedimientos de toma de decisión políticos alientan un debate libre, reflexivo e informado en el que todos los afectados pueden participar o están adecuadamente

${ }^{3}$ Como podrá notarse, la posición de Nino es similar a la defendida por Thomas Scanlon en su influyente artículo "Contractualism and Utilitarianism" (véase Scanlon 1982) y que luego el autor desarrolló en What we owe to each other (Scanlon 1999)

${ }^{4}$ Nino ofrece una caracterización del concepto de "argumento genuino" siguiendo a Jon Elster. Véase Nino (1997, p. 171 y ss.). 
representados, la deliberación política preserva, en cierto grado, las virtudes cognitivas del discurso moral. Las ideas adicionales asociadas a la "tesis epistémica" juegan un papel importante en el terreno político. Dado que la mayoría, cuando su decisión deriva de un proceso deliberativo adecuado, tiene una probabilidad más alta de arribar a la posición correcta, los individuos que creen que se ha cometido un error tienen una buena razón para obedecer las normas surgidas del proceso democrático aun cuando se encuentren seguros de que están en lo correcto en el caso particular en cuestión. Ello se debería a que en el largo plazo las mayorías tendrían más probabilidades de aproximarse a la verdad moral. Sin embargo, reconocer que los disidentes, por minoritarios que sean, pueden tener alguna probabilidad de estar en lo correcto es una buena razón para que las mayorías tengan en cuenta seriamente sus posiciones y permanezcan abiertos canales institucionales para el cuestionamiento y la revisión de las decisiones que surgieron del voto. Por otra parte, la idea de que los individuos son los mejores intérpretes de sus intereses apoya la tesis de que el proceso de deliberación debe ser lo más inclusivo posible y de que ningún grupo de supuestos expertos morales o de otro orden pueden legítimamente interpretar o reinterpretar los intereses de terceros sin incluirlos efectivamente en el proceso.

\section{2}

Nino presenta la "tesis epistemológica" como una alternativa a dos posiciones opuestas que vincula, respectivamente, a John Rawls y a Jürgen Habermas. ${ }^{5}$ La primera afirma que el conocimiento de la verdad moral se alcanza solo por medio de la reflexión individual. La segunda, por el contrario, que la discusión y la decisión colectiva ofrece el único acceso posible a la verdad moral. ${ }^{6}$ Ya conocemos la posición de Nino, una suerte de punto medio entre estos dos extremos que parecen, incluso a primera vista, muy poco plausibles. Para Nino la reflexión individual puede alcanzar la verdad moral o arribar a posiciones más plausibles que las que son alcanzadas, en algunos casos, incluso tras un proceso intersubjetivo de deliberación desarrollado en condiciones altamente

\footnotetext{
${ }^{5}$ Creo que ninguna de estas posiciones epistemológicas representan adecuadamente las posiciones de estos autores, pero no hay aquí espacio para discutir el punto, ni resulta relevante para la meta de la discusión.

${ }^{6} \mathrm{Al}$ igual que en la propuesta de Nino, tanto en el caso de Rawls como en el de Habermas se trataría de la "verdad moral" restringida al "área de la justicia" (véase Nino 1989, p. 105).
} 
favorables, es decir, condiciones que preservarían en un grado significativo el potencial cognitivo de dicho proceso. Sin embargo, la deliberación colectiva -mientras cumpla con ciertas reglas procesalessiempre tendrá, a la larga, más probabilidades de producir resultados correctos o cognitivamente superiores. Como vimos en la sección anterior, estas consideraciones tienen consecuencias políticas claras. Aunque un sujeto puede, a través de la reflexión individual, llegar a concebir soluciones superiores a las que resultan del proceso democrático, si dicho proceso presenta los rasgos apropiados, tendrá, en general, una probabilidad mayor de producir soluciones correctas. Como consecuencia de ello, aun sabiendo que la decisión mayoritaria es errónea, los individuos que quedan en minoría tienen razones morales -derivadas de consideraciones epistemológicas- para acatarla.

Pero, ¿es realmente plausible la argumentación a través de la cual Nino llega a estas conclusiones? El primer problema es que el escenario de confrontación entre las posiciones epistemológicas opuestas atribuidas a Rawls y Habermas, frente al cual la posición de Nino se presenta como una respuesta superadora, es muy poco plausible -dejando de lado la cuestión de si los filósofos citados suscribieron realmente estas tesis-. Imaginemos el desarrollo del proceso político en una democracia deliberativa cuyas instituciones encarnan ideas como las que defiende Nino. Frente a un conflicto, tras un proceso de deliberación libre, inclusivo, informado, etc., los representantes de los ciudadanos votan y la política P1 obtiene apoyo mayoritario. Los defensores de la política P2, la segunda opción más votada, piensan que el grupo mayoritario ha cometido un error: a su juicio $\mathrm{P} 1$ es moralmente inaceptable.

Si seguimos a Nino debemos describir la situación en los siguientes términos. La afirmación de que implementar P1 es la solución moralmente correcta o verdadera es el resultado de un proceso de deliberación intersubjetivo y de una votación en la que dicha posición obtuvo apoyo mayoritario. La creencia de que la implementación de P1 es la mejor alternativa puede ser falsa, pero tiene más probabilidades de ser verdadera que las otras alternativas que fueron analizadas. Por otra parte, la creencia de que P2 es la alternativa correcta, mientras que P1 es moralmente inaceptable, compartido, tras el proceso de deliberación, por muchos ciudadanos y sus representantes políticos, solo puede ser mantenido por dichos sujetos como el resultado de su juicio individual o del ejercicio de su capacidad de reflexión personal: como consecuencia de ello, tiene una probabilidad menor de ser correcto o verdadero, aun cuando esa posibilidad no puede ser excluida. Esta descripción de la situación resulta, sin embargo, muy poco plausible. Una interpretación más 
razonable del caso afirmará que tanto la afirmación de que P1 es la política correcta y P2 una opción inadecuada o moralmente inaceptable como la afirmación contraria tienen el mismo estatus intersubjetivo. Como resultado del proceso de discusión libre, inclusivo e informado en que se revisaron críticamente diversas alternativas, un grupo de individuos llegó a la conclusión de que P1 era la mejor opción y otro grupo a la conclusión de que P2 era la mejor opción. El primer grupo resultó más numeroso que el segundo. Los que pierden la votación no son individuos aislados que se apoyan meramente en su juicio solitario, son también una comunidad de argumentación en la que hay deliberación y, supuestamente, progreso cognitivo. De este modo el contraste entre juicios respaldados en la reflexión individual y juicios respaldados por un proceso de deliberación intersubjetiva -que poseerían un potencial cognitivo superior al del primer tipo de juicios- desaparece y, con él, la premisa de la que depende la plausibilidad de la línea central de la argumentación de Nino. Los agentes que defienden P2 no tienen por qué aceptar que P1 tiene mayor probabilidad de ser la solución correcta como consecuencia del hecho de que su creencia en la superioridad de P2 deriva, supuestamente, de su juicio individual, mientras que las creencias de los defensores de $\mathrm{P} 1$ son el resultado de un proceso colectivo o intersubjetivo de deliberación. Los defensores de P2 pueden aducir que dicha política está, por lo menos, en igualdad de condiciones en ese respecto: también es el resultado de un proceso de deliberación colectiva y no el resultado de una mera agregación de juicios individuales aislados.

Podría argumentarse, contra la objeción precedente, que he pasado por alto una distinción importante en la propuesta de Nino. ${ }^{7}$ Nino distinguiría dos instancias que contribuyen al valor epistémico de las decisiones democráticas: 1) el que surge de la deliberación desarrollada en condiciones apropiadas y 2) el que deriva de la implementación de un procedimiento electoral regido por la regla de la mayoría. La objeción formulada líneas atrás parece afectar solo al punto 1). Sin embargo, quedaría en pie 2) cuya justificación deriva del "teorema del jurado", formulado originalmente por Condorcet.

Presentado en forma simple -no disponemos de espacio para una caracterización detallada-, el teorema del jurado afirma que la probabilidad de que una decisión correcta sea tomada en un contexto determinado aumenta drásticamente si es apoyada por un número elevado

7 Agradezco a un/a evaluador/a anónimo/a de la revista Análisis Filosófico esta objeción y otras recomendaciones que han sido tomadas en cuenta en la redacción final del presente artículo. 
de agentes cuya competencia epistémica es superior al azar. Es decir, por agentes que tienen más de un cincuenta por ciento de probabilidades de escoger la alternativa correcta. El teorema se aplica, en principio, a contextos en que se debe elegir entre dos cursos de acción alternativos. Si el número de votantes a favor de una de las alternativas es grande y todos poseen una probabilidad levemente superior al cincuenta por ciento de acertar, el resultado es prácticamente la infalibilidad. ${ }^{8}$ De forma correlativa, cuando la competencia epistémica de los individuos que confirman un grupo numeroso es inferior al azar, la conclusión del teorema es la certeza o la cuasi certeza de que escogerán la opción errónea.

Apelar al teorema del jurado parece, sin embargo, poco prometedor, dado que su aplicabilidad a contextos políticos ha sido blanco de objeciones significativamente plausibles. ${ }^{9}$ Una de las dificultades más importantes, siguiendo a David Estlund, consiste en que no parece haber forma de justificar de un modo públicamente aceptable el presupuesto, fundamental para el teorema, de que los agentes que forman parte del grupo mayoritario tengan, en promedio, una probabilidad de tomar la decisión correcta mayor al azar o a la aplicación de un procedimiento aleatorio. Como afirma Estlund, tal cosa podría ocurrir en ciertos casos y, como consecuencia de ello, los procedimientos democráticos podrían resultar "virtualmente infalibles" para resolver cuestiones acerca del bien común. Sin embargo el problema es que, aun en ese caso, "parece imposible establecer [tal cosa] públicamente sin un acceso independiente a la verdad" (Estlund 1993, p. 93). Si no disponemos de tal acceso independiente a la verdad -relativo a las cuestiones fácticas, morales, etc. que están en juego-no podremos saber con qué frecuencia un individuo o un grupo acierta o se equivoca en sus juicios. Por lo tanto, no podemos sacar ninguna conclusión acerca de la competencia epistémica del agente o agentes involucrados. Consideremos el siguiente ejemplo para clarificar el problema. Supongamos que P1 y P2 son dos conjuntos de medidas económicas alternativas e incompatibles, cuya meta es superar una crisis que afecta a una nación en un momento determinado. $\mathrm{P} 1$ producirá una salida rápida de la crisis imponiendo elevados costos a un sector minoritario y vulnerable de la población. Sin embargo, su resultado esperable es un incremento significativo del

${ }^{8}$ Como señala Estlund, si los miembros de un grupo de 250 personas tienen, cada uno, una probabilidad de acertar de 0,51 -es decir, mínimamente superior al azar-su probabilidad grupal promedio será de 0,62 , pero un grupo de 10.000 personas con esa misma probabilidad individual tendrá una probabilidad grupal cercana a la infalibilidad, 0,98. (Véase Estlund 1994, p. 131).

${ }^{9}$ Véase, por ejemplo, Gaus (1997, p. 150) y Estlund (1993, pp. 93-94 y 2008, pp. 223236). 
bienestar de la mayoría. P2 también ofrece una salida a la crisis, aunque más lenta y con un resultado global, en términos del nivel de recuperación económica, inferior al ofrecido por P1. El punto a favor de P2 es que involucra un esquema mucho más igualitario en el reparto de los costos que deberán pagar los ciudadanos para salir de la crisis. Supongamos que las razones aducidas por los defensores de P1 para sostener que es moralmente correcta y P2 incorrecta, dependen de la apelación a alguna variante de la teoría utilitarista. La corrección moral de una acción depende de que esta constituya, entre las alternativas disponibles, la que maximiza la suma neta de bienestar de los afectados (sin importar cómo se distribuye ese bienestar entre ellos). Los defensores de P2 sostienen que P1 es moralmente inaceptable y las razones de fondo por las que lo afirman podrían ser descritas como "kantianas". La idea en este caso es que no resulta moralmente aceptable imponer coercitivamente costos a algunos individuos para mejorar la situación global de la sociedad, dado que se estaría vulnerando el principio de igual dignidad de las personas. Los miembros de cada grupo deliberan entre sí en distintos foros y luego deliberan con los miembros del grupo que sostiene la posición antagónica. En ese proceso puede suponerse -siguiendo a Nino y a los defensores de teorías similares- que debería mejorar la calidad epistémica de los argumentos que termina aduciendo cada grupo a favor de su posición. Supongamos que no hay consenso: un grupo insiste en aducir argumentos de raigambre kantiana a favor de $\mathrm{P} 2$ y el otro grupo insiste en aducir argumentos de raigambre utilitarista a favor de P1. Se pasa a votar y P1 obtiene el $60 \%$ de los votos.

Aun suponiendo que fuera cierto que hubo una mejora epistémica debida a la deliberación en la justificación de ambas políticas, ¿cómo saber si la competencia epistémica promedio de los votantes era superior o inferior al cincuenta por ciento de probabilidades de acertar (es decir, a 0,5)? Si los agentes que identificamos como kantianos tienen razón en que es moralmente inaceptable imponer cargas severas a una minoría para maximizar el bienestar general, entonces, dado el resultado de la votación, es perfectamente razonable afirmar que había más probabilidades de que la política correcta fuera implementada tomando la decisión al azar, lanzando una moneda, que votando. Ello equivale a decir que la competencia epistémica promedio de los votantes de P1, que obtuvieron el $60 \%$ de los votos, era inferior a 0,5. El único modo refutar esta conclusión parece requerir demostrar que el principio de utilidad es el canon moral correcto y que sus objetores se equivocan. Pero eso no puede hacerse diciendo que la mayoría lo aprueba y ha votado P1 motivado por la creencia de que es correcto. 
Ahora bien, supongamos que la objeción precedente puede ser refutada y es posible saber el nivel de competencia epistémica promedio de un individuo o de un grupo. En ese caso, contra lo que pensaba Nino, puede sostenerse que el teorema del jurado es una amenaza para los defensores de una democracia igualitaria. Como señala Estlund

Si fuera posible saber si la competencia individual promedio está por debajo de 0,5 , parece viable considerar que se podrá saber algo acerca de quién es más competente que quién. Dependiendo de los números, hay muchas circunstancias en que la competencia del grupo resultaría incrementada privando del voto a los menos competentes. En primer lugar, aquellos cuya competencia es inferior a 0,5 no contribuyen en nada, en ninguna circunstancia, a la competencia del grupo. En segundo lugar, incluso aquellos que están por encima de 0,5 pueden, dependiendo de las circunstancias, causar un deterioro a la competencia del grupo dado que no son tan competentes como los otros. En otra palabras, si tenemos suficiente información como para saber que el modelo condorcetiano puede ser aplicado, muy probablemente tendremos suficiente información para limitar el sufragio a un subconjunto acotado de ciudadanos, lo cual no constituiría otra cosa que una élite autoritaria. (Estlund 1993, pp. 93-94).

Podemos concluir que la apelación al teorema del jurado o bien no resulta de ninguna utilidad para defender la concepción epistémica del valor de la democracia propuesta por Nino o bien constituye una amenaza potencial contra dicha concepción.

El ejemplo de las políticas P1 y P2 permite identificar un problema adicional en la propuesta de Nino. Como vimos, se trataba de la contraposición entre una política económica, P1, cuya justificación depende para sus defensores de consideraciones utilitaristas y una política económica alternativa, P2, que presupone el compromiso con una interpretación kantiana de lo que requiere el respeto igualitario por todos los miembros de la comunidad. Afirmar que P1 "depende" de consideraciones utilitaristas y P2 de consideraciones kantianas puede dar lugar a confusiones. Es posible que esas políticas pudieran ser justificadas aduciendo a otro tipo de razones, morales o de otro orden (prudenciales, por ejemplo). Estoy suponiendo que los defensores de P1 consideran que la superioridad de dicha política se debe a que 
constituye una aplicación adecuada de los principios utilitaristas y que lo mismo ocurre con los defensores de P2: su superioridad radica para ellos en razones morales más generales que hemos caracterizado como "kantianas". Supongamos, adicionalmente, que en el debate, cada grupo critica y rechaza los fundamentos más generales que aduce el grupo adversario para defender su propuesta. Los defensores de P2, por ejemplo, sostienen que maximizar la suma neta de bienestar no puede constituir un criterio de justicia adecuado porque podría justificar la violación de derechos fundamentales de los ciudadanos. Los defensores de P1 afirman, por ejemplo, que las ideas kantianas en que apoyan sus adversarios la defensa de su posición constituyen abstracciones vacías o que dependen de intuiciones altamente controvertidas acerca de la noción de dignidad personal. Ahora bien, los debates entre utilitaristas y kantianos son, por así decirlo, clásicos tanto en ética como en filosofía política. Sin embargo, no parece tener sentido suponer que una votación entre académicos, tras un debate libre y abierto, pueda ofrecer razón alguna para concluir que una de las posiciones, la que obtenga en un momento dado el apoyo de la mayoría, es la correcta o la más cercana a la verdad. Las votaciones pueden ser una herramienta adecuada, en algunas instituciones académicas, para tomar ciertas decisiones prácticas o políticas (en el sentido especial que adquiere ese término en este contexto). Podría utilizarse, por ejemplo, para decidir a qué grupo de académicos se otorga un subsidio para desarrollar una investigación o si se destina un nuevo edificio a la enseñanza de grado o de postgrado. Pero no resulta razonable pensar que la deliberación combinada con votación sea una herramienta para evaluar el potencial cognitivo de distintas posiciones filosóficas. No hay duda de que las instituciones académicas y los debates entre filósofos difieren en aspectos significativos de los debates en la arena política. Sin embargo, las consideraciones precedentes parecen resultar pertinentes en ambos casos. Si la deliberación entre filósofos que defienden posiciones utilitaristas y kantianas, combinada con algún mecanismo que permita determinar quienes están en mayoría luego de ese proceso de intercambio de razones, no permite extraer una conclusión acerca de qué posición es la correcta, una combinación entre deliberación y votación no permitirá tampoco determinar si P1, una política cuya aceptabilidad moral depende -en el ejemplo propuesto- de consideraciones utilitaristas, es cognitivamente superior a P2, que depende de consideraciones de tipo kantiano. Es perfectamente plausible decir que en un escenario político, al igual que en el ejemplo de la función de las votaciones en instituciones académicas, la combinación entre deliberación y votación tiene también una función práctica, no cognitiva, confiere legitimidad a la decisión de 
implementar, por ejemplo, P1, y ello se debe a que todos aceptan -es decir, han decidido aceptar- desde el principio una práctica entre cuyas reglas está respetar la voluntad mayoritaria si esta es compatible con ciertos derechos fundamentales ${ }^{10} \mathrm{y}$ cuando el proceso político ha permitido a todos, en el grado más elevado que se pueda alcanzar en la práctica, exponer sus puntos de vista e intentar obtener el apoyo del grupo más numeroso.

Es interesante señalar que Nino afirma que el valor epistémico del procedimiento democrático

no se aplica, por ejemplo, al contexto de temas científicos o, en general, fácticos, incluso cuando el proceso de discusión pueda implicar un beneficio para estos dominios. No hay ningún presupuesto a favor de los juicios científicos y fácticos en general solo porque ellos sean apoyados por una opinión mayoritaria. Lo mismo se aplica a asuntos religiosos o filosóficos. (Nino 1997, p. 182).

Esta declaración es, como podrá notarse, extremadamente problemática dado que confirma y, podríamos decir, generaliza la objeción precedente. La justificación de la política económica de un estado, especialmente en momentos de crisis, involucrará normalmente la apelación a consideraciones "fácticas", "científicas" y, en muchos casos, a un trasfondo moral "filosófico" como el que citamos: concepciones incompatibles -al menos en principio- de la equidad (como vimos en el ejemplo anterior, posiciones consecuencialistas o deontológicas, posiciones que dan prioridad a la libertad sobre la igualdad o viceversa, etc.). Lo mismo ocurrirá en una amplia gama de políticas fundamentales para el Estado: la política inmigratoria, de salud pública, problemas vinculados con el medioambiente, política energética, etc. Por otra parte, algunos sujetos defenderán la idea de que la apelación a consideraciones religiosas no puede ser reivindicada como un recurso argumentativo legítimo en la política democrática. Si se considera que la deliberación, como afirma Nino, no posee valor epistémico cuando estos elementos entran en juego,

${ }^{10}$ Nino suscribe esta concepción liberal. Según sostiene: "los derechos [individuales básicos] no se derivan del proceso democrático sino que surgen de reflexiones efectuadas sobre los presupuestos de nuestra práctica de la discusión moral. Una vez que descubrimos estos derechos, su función parece ser precisamente la de limitar la operación del proceso democrático a través de la descalificación de decisiones colectivas que los ignoran. [...] Esta es la intuición básica detrás de la idea de que cuando el constitucionalismo es sumado a la democracia, hay decisiones democráticas que están impedidas por el reconocimiento liberal de los derechos" (Nino 1997, p. 95). 
parece realmente muy poco aquello que puede ser objeto de un debate fructífero. El objeto de aplicación de la teoría de Nino se desvanece. Probablemente la mayoría de las cuestiones políticas relevantes o, al menos, un número muy significativo de ellas, no es fácilmente separable de tesis científicas controvertidas, visiones del mundo que pueden tener componentes religiosos o tesis morales o relativas a la naturaleza de la justicia social que pueden ser descritas como filosóficas. Si seguimos a Nino, basta con que las posiciones políticas de un agente o un grupo estén fundamentadas en ese tipo de consideraciones para tenga todo derecho a negar que el debate y la formación de una mayoría electoral adversa a la posición que defiende diga algo acerca de la probabilidad de que dichas posiciones sean acertadas o erradas. Si estos sujetos deben obedecer las políticas estatales que se implementen como consecuencia de ese proceso, ello no puede tener ninguna relación con un reconocimiento de calidad epistémica de dichas políticas.

\section{4}

En Liberalismo político Rawls rechazó con claridad la apelación a consideraciones epistémicas para dar cuenta de la legitimidad política: esta dependía en forma directa de la razonabilidad y racionalidad -en el sentido técnico que le daba el autor a estos términos- de la norma, no de que fuera la solución basada en la concepción moral, religiosa o filosófica correcta o verdadera del problema. ${ }^{11}$ Tanto la estrategia de Rawls en particular como otras posiciones conceptualmente similares acarrean dificultades que no es posible discutir en detalle aquí. ${ }^{12}$ Sin embargo, para terminar, quisiera ofrecer el esbozo de un argumento adicional que se encuadra en una perspectiva afín al liberalismo político ${ }^{13}$ sobre la naturaleza de la legitimidad política. Dicho argumento volverá más claras las razones por las que pienso que Nino cometió un error al

11 Una de los rasgos característicos del liberalismo político rawlsiano consiste en la tesis de que la razonabilidad, y no la verdad, constituye el criterio de corrección de las concepciones políticas de la justicia (véase Rawls 1993, p. 127). Según el autor, el liberalismo político "no usa [...] ni niega el concepto de verdad; tampoco pone en cuestión ese concepto, ni podría decir que el concepto de verdad coincide con su idea de lo razonable. Ocurre más bien que, dentro de sí misma, la concepción política prescinde del concepto de verdad" (Rawls 1993, p. 94).

12 Para una crítica de la posición de Rawls, véase Cohen (2009). Para una defensa de la posición rawlsiana, véase Garreta Leclercq (2010).

${ }^{13}$ He defendido en forma más detallada esta concepción en Garreta Leclercq (2012) y (2013). Debo aclarar que mi posición, aunque aspira a constituir una forma de liberalismo político, se aparta en aspectos importantes de la propuesta de Rawls. 
defender un enfoque epistémico del valor de la democracia y la deliberación pública.

Tanto Nino como una larga tradición dentro de la filosofía política occidental han tendido a pasar por alto algunas particularidades del razonamiento práctico que lo hacen significativamente diferente del razonamiento teórico (o de ciertas concepciones "intelectualistas" muy influyentes del razonamiento teórico). Muchos piensan que las únicas consideraciones que cuentan para que un sujeto esté justificado a creer que $p$ son siempre de orden evidencial o razones relativas, de un modo plausible, a la verdad o falsedad de que $p$. Aunque cuestionada por algunos epistemólogos (no parece haber nada en la filosofía que esté exento de controversia) esta posición que podríamos llamar "evidencialismo" o "purismo" es altamente plausible. ${ }^{14}$ No hace falta cuestionarla, sin embargo, para tomar conciencia de que estar justificados a creer que $p$ no ofrece necesariamente una justificación moral ${ }^{15}$ para obrar sobre la base de dicha creencia en cualquier contexto. Imaginemos un ejemplo simple. En el escenario A, el sujeto S1 se encuentra en una estación de tren que conoce bastante bien. Hay dos trenes a punto de salir. Los dos se dirigen al destino al que planea viajar S1. La diferencia es que uno de los trenes hace muchas menos paradas y tarda menos de la mitad del tiempo en arribar al destino elegido por el agente. S1 escucha a un empleado del ferrocarril confirmar a otro pasajero que el tren rápido es el que se encuentra en el andén 1 y luego lee el anuncio en un cartel. S1 ha constatado en el pasado que ese tipo de información es confiable, pero no exenta de un nivel significativo de probabilidad de error. Ahora bien, el escenario B es idéntico al precedente en varios aspectos: el sujeto S2 tiene el mismo conocimiento de la terminal de trenes y su funcionamiento que S1 y la misma información sobre cuál es, aparentemente, el tren rápido. S1 y S2 creen, sobre la base de la misma evidencia, que el tren del andén 1 ofrece el servicio con menos paradas. Sin embargo, hay una diferencia práctica en la situación de ambos sujetos. Si S1 está cometiendo un error ello no acarrea ninguna consecuencia grave ni para él ni para terceros. Por el contrario, si S2 está cometiendo un error y, como consecuencia de ello llega más tarde a su destino, otro sujeto sufrirá un daño grave e irreversible. Algunos epistemólogos han sostenido que en casos como el citado un observador estaría justificado a afirmar que S1

${ }^{14}$ Para una defensa del evidencialismo véase Conee y Feldman (2005).

$15 \mathrm{El}$ caso moral es el que más nos interesa aquí, pero lo mismo puede decirse en relación con consideraciones estratégicas o instrumentales puramente egoístas o moralmente neutras. 
sabe que $p$ ("el tren del andén 1 es el rápido"), pero no estaría justificado a afirmar que $\mathrm{S} 2$ tenga tal conocimiento. ${ }^{16}$ Otros sostienen tesis más fuertes, por ejemplo, que $\mathrm{S} 1$ sabe que $p$, mientras que $\mathrm{S} 2$ no sabe que $p$, aun cuando ambos dispongan de la misma evidencia. ${ }^{17}$ Esta última tesis constituye, claramente, un rechazo de una posición clásica y dominante en la epistemología analítica, el evidencialismo, que consiste en la tesis de que si dos sujetos disponen de la misma evidencia para afirmar una creencia o bien los dos están igualmente justificados en sostenerla o bien ninguno lo está. No corresponde aquí tomar posición acerca de la plausibilidad del evidencialismo. El punto interesante es que desde una perspectiva moral, centrada en el problema no de la justificación epistémica de creencias sino de la justificación de acciones, aun aceptando el evidencialismo, resulta perfectamente plausible decir que mientras S1 está justificado a creer que $p$ y actuar sobre la base de esa creencia -lo que implica subir al tren del andén 1 sin tomar recaudos adicionales-, S2 no está justificado a actuar, aunque esté igualmente justificado que $\mathrm{S} 1$ a suscribir dicha creencia. El punto es que dado el costo del error, aun cuando S2 crea tan justificadamente como S1 que $p$, no está justificado a actuar sobre la base de esa creencia: hay demasiado en juego y podría estar cometiendo un error de consecuencias muy graves. Si S2 actuara de igual modo que en una situación normal, como la que ejemplifica la situación de S1, en la que tomar el tren que tarda más en llegar a destino no tiene consecuencias graves, y de hecho cometiera un error, sería objeto de censura y considerado responsable, al menos en parte, de las desastrosas consecuencias de sus acciones. En escenarios como aquel en que se encuentra S2 la situación se podría resolver, al menos en muchos casos, de forma sencilla: buscando información adicional, al punto en que resultara irrazonable decir que el agente es responsable si se produjera, igualmente, un resultado indeseable. El problema es que hay muchas situaciones en las que "investigar más", "deliberar", o apelar a procedimientos o acciones cuyo supuesto resultado es alguna clase de progreso cognitivo no resultan suficientes para justificar la acción. Imaginemos que un médico le informa a un paciente que hay dos tratamientos posibles para la enfermedad ocular que ha contraído. En primer lugar, medicación que producirá una mejora moderada de su enfermedad. En segundo lugar, una operación que la curaría por completo. La cuestión es que la medicación no puede producir ningún daño al

16 Esta parece la idea básica de los contextualistas como Stewart Cohen o Keith DeRose (véase Cohen 1999 y DeRose 1992).

17 Esta posición ha sido defendida, por ejemplo, por Fantl y McGrath (2007). 
paciente, mientras que la operación, en un número muy bajo de casos, puede agravar el cuadro en forma drástica e irreversible, dejándolo ciego. El médico está seguro de que el paciente debe operarse, que es la mejor alternativa, pero el paciente no quiere correr el riesgo, por pequeño que sea. Es fácil saber que aunque el médico estuviera en lo correcto y la mejor decisión fuera que el paciente se someta a la operación, no está moralmente justificado a actuar sobre la base de esas creencias contra la voluntad de dicho agente. El médico puede hacer un simple experimento mental para llegar a esa conclusión. Si engañara al paciente y lo operara contra su voluntad y la operación saliera mal, no por un error en su ejecución, sino porque el paciente pertenece a ese pequeño y previamente indetectable porcentaje de individuos que no responde bien a esa terapia, sería plenamente responsable del daño sufrido por dicho individuo. Estar justificado a actuar es, por el contrario, no ser responsable por las consecuencias indeseables de una acción cuando las cosas salen mal. Por supuesto, el médico no ganaría nada investigando más, no hay tiempo para eso, está actuando sobre la base de los conocimientos establecidos en su disciplina en un momento determinado, no es ni siquiera un investigador. La situación cambiaría si mediara el consentimiento informado del paciente: ello relevaría de responsabilidad al médico en caso de que la operación saliera mal. La solución no es epistémica, sino práctica. El problema en este tipo de casos es que, en contextos donde hay mucho en juego y el bienestar de terceros puede ser severamente dañado en caso de error, nadie tiene derecho a decidir unilateralmente correr el riesgo por los afectados. La calidad epistémica de la creencia de que un curso de acción es la mejor alternativa, si hay un nivel significativo de probabilidad de error y ese resultado implica un alto costo en el bienestar de terceros, nunca es suficiente para estar justificado a actuar en forma unilateral, sin la mediación de procedimientos de autorización previamente estipulados y aceptados por los afectados o en forma generalizada.

La arena política ofrece situaciones similares en aspectos importantes. En el caso de los conflictos políticos que afectan a los bienes primarios -en el sentido que da Rawls al término- de los que dependen que los ciudadanos tengan la posibilidad de llevar adelante una vida valiosa y con sentido, se aplican consideraciones análogas, al menos en algunos puntos importantes, al caso precedente. Supongamos que hay dos modelos o planes económicos, A y B, muy diferentes, que son propuestos y defendidos por economistas y políticos de distintas orientaciones ideológicas. Economistas y políticos asesorados por economistas defienden en el foro público dichos planes. Cada bando sostiene que su propuesta 
es la mejor: que la alta probabilidad de que produzca los mejores resultados está justificada sobre la base de consideraciones teóricas y empíricas sólidas. Supongamos que hay un amplio debate en distintos foros públicos. Ahora bien, el fracaso del plan económico A, previsiblemente, produciría un daño muy severo en un sector minoritario de la población, por ejemplo, los trabajadores retirados que cobran jubilaciones más bajas. El fracaso de B produciría un empeoramiento de la calidad de vida de la población, mucho menos agudo que el producido por A sobre los jubilados de menores recursos, pero que afectaría a un sector mucho más numeroso. Hay que aceptar que en cuestiones complejas y en las que intervienen tantas variables imprevisibles, la probabilidad de error es siempre alta. Sin embargo, los defensores de ambas políticas pueden estar sólidamente justificados en creer que la alternativa que defienden tiene más probabilidades de éxito y es, además, la solución más justa. Investigar más o deliberar más no cambia las cosas. Es importante notar que la razón por la que puede ser legítimo que el gobierno implemente una de esas políticas, A o B, contra la opinión de parte de la población, no depende de la calidad epistémica de la política elegida -ambas pueden tener una alta calidad epistémica tras el debate y debido a la intervención de especialistas-, sino del hecho de que la decisión haya surgido de mecanismos de autorización para la acción apropiados: deliberación en la esfera pública y en los organismos políticos que toman las decisiones, justificación pública de esas decisiones, votación de los representantes de los ciudadanos -en caso de que la decisión dependa, al menos en parte, del poder legislativo-, posibilidad de crítica, revisión y modificación de las decisiones, etcétera. Los que pierden no dan su consentimiento directo, como el paciente del ejemplo anterior, sino aceptan el conjunto de procedimientos preexistentes de autorización y la legitimidad de la decisión cuando dichos procedimientos se han respetado. Como consecuencia de ello, no puede decirse que los que ganan hayan decidido unilateralmente correr el riego por sus conciudadanos. La decisión está justificada aunque la política fracase. Cuando hay mucho en juego, desacuerdo persistente y razonable -es decir, cuando todas las partes pueden aducir razones plausibles en favor de sus posiciones- y la probabilidad de error es significativa, como ocurre con la mayoría de las decisiones políticas importantes en las democracias contemporáneas, la cuestión de la calidad epistémica de las decisiones no juega un papel decisivo, contra lo que pensaba Nino, en la legitimidad moral de las decisiones. Por el contrario, esa legitimidad depende del consenso o la aceptación -aunque sea tácita- sobre qué procedimientos deben satisfacerse para que esas decisiones que no pueden concitar consenso 
sean, no obstante, legítimas. Esas decisiones no son normalmente actos de voluntad ciegos, requieren de justificación sobre la base de razones dotadas de valor cognitivo, pero no dejan de tener un estatus centralmente práctico.

\section{Conclusión}

En la sección inicial del artículo se reconstruye el núcleo de la argumentación ofrecida por Nino para defender su concepción deliberativa de la democracia. Luego, en las secciones 2 y 3 , se desarrollan dos objeciones contra dicha concepción. En primer lugar, se intenta demostrar que la contraposición planteada por Nino entre las probabilidades que deberían atribuirse al acceso individual a la verdad moral, por una parte, y al acceso intersubjetivo o deliberativo, por otra, no pueden jugar el papel que dicho autor les atribuye en su propuesta. Aunque fuese cierto que una deliberación desarrollada en condiciones apropiadas y que propiciara la participación de todos los afectados tuviera más probabilidades de producir resultados correctos que la reflexión individual, eso no probaría que las decisiones mayoritarias poseyeran ese mismo rasgo. Como vimos, ello se debe a que el debate político tiene lugar siempre, o normalmente, entre grupos que acuñan sus posiciones de forma igualmente dialógica e intersubjetiva. Las posiciones de las mayorías y de las minorías tienen, al menos en principio, la misma presunción de calidad -o falta de calidad- epistémica. Presentar a la posición mayoritaria como el resultado de un procedimiento de deliberación intersubjetiva y a la minoritaria como la adición de un conjunto coincidente de conclusiones surgidas de la reflexión individual resulta completamente arbitrario. Sin embargo, solo ese supuesto permitiría concluir, siguiendo la lógica del planteo de Nino, que la posición mayoritaria tiene una probabilidad mayor de ser correcta o verdadera. Se analizó también la viabilidad de neutralizar esta crítica mediante la apelación al teorema del jurado y se presentaron objeciones, conocidas en la literatura sobre el tema, contra la plausibilidad de esta estrategia. La segunda objeción apuntó a demostrar que la propuesta de Nino falla en tomar en cuenta las consecuencias de lo que Rawls denominó el "hecho del pluralismo" razonable. Gran parte del debate político apela a supuestos conocimientos científicos, concepciones filosóficas, morales o religiosas en las que no tiene sentido esperar que el debate, aun llevado adelante en las condiciones más favorables para el progreso cognitivo, permita arribar a concusiones firmes acerca de qué punto de vista es el correcto. Dado que Nino acepta que la deliberación pública no tiene fuerza cognitiva en esas 
materias, resulta muy difícil entender cuándo la deliberación podría tener el papel que le asigna su teoría. Según vimos, si es correcto que la deliberación -como afirma Nino- no cumple una función cognitiva en cuestiones filosóficas -que pueden incluir muchas cuestiones morales relevantes para la justicia social- o religiosas, basta que un grupo minoritario sostenga que las políticas que defiende obtienen su justificación en este tipo de consideraciones para que esté autorizado a negar que la posición mayoritaria, surgida de la deliberación y el voto, tenga una probabilidad mayor de ser correcta. Como consecuencia de ello queda neutralizada la tesis central de Nino: la minoría no tiene razones morales para obedecer las políticas que surgen del proceso democráticodeliberativo.

Por último, en la sección 4, se ofreció el esbozo una concepción alternativa a la propuesta por Nino acerca de la naturaleza de la legitimidad política. Desde esta perspectiva el sentido del proceso democrático y el valor de la deliberación son concebidos a la luz del hecho de que las consideraciones epistémicas resultan insuficientes para justificar la acción del Estado en todos los casos en que dicha acción puede tener un impacto significativo sobre el bienestar de los ciudadanos. Esta concepción postula una tesis opuesta a la de Nino: la razón de ser de la deliberación democrática no es, en última instancia, su valor epistémico, sino, por el contrario, el hecho de que en contextos donde hay mucho en juego las virtudes epistémicas de una creencia o un conjunto de creencias no son nunca suficientes por sí mismas para justificar la acción coercitiva del Estado.

\section{Bibliografía}

Cohen, J. (2009), “Truth and Public Reason”, Philosophy \& Public Affairs, 1 , pp. $2-42$.

Cohen, S., (1999), "Contextualism, Skepticism, and the Structure of Reasons", Philosophical Perspectives 13: Epistemology, pp. 57-89.

Conee, E. y Feldman, R. (2005), Evidentialism, Oxford, Oxford University Press.

DeRose, K. (1992), "Contextualism and Knowledge Attributions", Philosophy and Phenomenological Research, 52(4), pp. 913-929.

Estlund, D. (1993), "Making Truth Safe for Democracy", en Copp, D., Hampton, J. y Roemer, J. (eds.), The Idea of Democracy, Cambridge, Cambridge University Press, pp. 71-100.

- (1994), "Opinion Leaders, Independence and Condorcet's Jury Theorem", Theory and Decision, 36(2), pp. 131-162. 
Estlund, D. (2008), Democratic Authority, Princeton, Princeton University Press.

Fantl, J. y McGrath, M. (2007), "On Pragmatic Encroachment in Epistemology", Philosophy and Phenomenological Research, LXXV(3), pp. 558-589.

Garreta Leclercq, M. (2010), "Liberalismo político, justificación pública y verdad", en Doxa. Cuadernos de Filosofía del Derecho, 33, pp. 215238.

— (2012), "Liberalismo político y reciprocidad: justificación epistémica de creencias versus justificación moral de acciones", Isegoría. Revista de Filosofía Moral y Política, 46, pp. 279-294.

- (2013), "Democracy and Deliberation: Two Models of Public Justification", en Revista Latinoamericana de Filosofía Política (RLFP), II(4), pp. 1-18.

Gaus, G. (1997), "Does Democracy Reveal the Will of the People? Four Takes on Rousseau", Australasian Journal of Philosophy, 75(2), pp. 141-162.

Nino, C. S. (1989), El constructivismo moral, Madrid, Centro de Estudios Constitucionales.

— (1997), La constitución de la democracia deliberativa, Barcelona, Gedisa.

Rawls, J. (1993), Political Liberalism, Nueva York, Columbia University Press.

Scanlon, T. (1982), "Contractualism and Utilitarianism", en Sen, A. y Williams, B. (eds.), Utilitarianism and Beyond, Cambridge, Cambridge University Press.

— (1999), What we owe to each other, Cambridge, The Belknap Press of Harvard University Press.

Recibido el 5 de octubre de 2014; aceptado el 1 de diciembre de 2014. 